\title{
Morbid Obesity: Increased Risk of Failure After Aseptic Revision TKA
}

\author{
Chad D. Watts MD, Eric R. Wagner MD, \\ Matthew T. Houdek MD, David G. Lewallen MD, \\ Tad M. Mabry MD
}

Received: 25 November 2014/ Accepted: 24 March 2015/Published online: 7 April 2015

(C) The Association of Bone and Joint Surgeons (B) 2015

\begin{abstract}
Background Patients with obesity are known to have a higher risk of complications after primary TKA; however, there is a paucity of data regarding the effects of obesity with revision TKAs.

Questions/purposes We asked the following questions : (1) Are patients with morbid obesity (BMI $\geq 40 \mathrm{~kg} / \mathrm{m}^{2}$ ) at greater risk for repeat revision, reoperation, or periprosthetic joint infection (PJI) compared with patients without obesity $\left(\mathrm{BMI}<30 \mathrm{~kg} / \mathrm{m}^{2}\right.$ ) after an index revision TKA performed for aseptic reasons? (2) Do patients who are not obese achieve higher Knee Society pain and function scores after revision TKA for aseptic reasons?

Methods We used a retrospective cohort study with 1:1 matching for sex, age ( \pm 3 years) and date of surgery ( \pm 1 year) to compare patients with morbid obesity with patients without obesity with respect to repeat revision, reoperation, and PJI. Using our institution's total joint
\end{abstract}

The institution of all authors has received, during the study period, funding from Zimmer (Warsaw, IN, USA), Stryker (Mahwah, NJ, USA), DePuy (Warsaw, IN, USA), and Biomet (Warsaw, IN, USA). One author certifies that he (DGL), or a member of his immediate family, has or may receive payments or benefits, during the study period, an amount of USD 100,001 to USD 1,000,000 from Zimmer, Inc.

All ICMJE Conflict of Interest Forms for authors and Clinical Orthopaedics and Related Research ${ }^{\mathbb{R}}$ editors and board members are on file with the publication and can be viewed on request. Each author certifies that his or her institution approved the human protocol for this investigation and that all investigations were conducted in conformity with ethical principles of research.

C. D. Watts, E. R. Wagner, M. T. Houdek,

D. G. Lewallen, T. M. Mabry ( $₫)$

Department of Orthopedic Surgery, Mayo Clinic, 200 First Street

S.W., Rochester, MN 55905, USA

e-mail: mabry.tad@mayo.edu registry, we identified 1291 index both-component (femoral and tibial) aseptic revision TKAs performed during a 15-year period (1992-2007). Of these, 120 revisions were in patients with morbid obesity (BMI $\geq 40 \mathrm{~kg} /$ $\mathrm{m}^{2}$ ) and 624 were in patients with a BMI less than $30 \mathrm{~kg} /$ $\mathrm{m}^{2}$. We then considered only patients with a minimum 5year followup, which was available for $77 \%$ of patients with morbid obesity and $76 \%$ of patients with a BMI less than $30 \mathrm{~kg} / \mathrm{m}^{2}$ ( $\left.\mathrm{p}=0.84\right)$. All patients with morbid obesity who met criteria were included (morbid obesity group: $\mathrm{n}=93$; average followup, 7.9 years) and compared with a matched cohort of patients with a BMI less than $30 \mathrm{~kg} / \mathrm{m}^{2}$ (nonmorbid obesity group: $\mathrm{n}=93$; average followup, 7.3 years). Medical records were reviewed to gather details regarding complications and clinical outcomes.

Results Overall, patients with morbid obesity had an increased risk of repeat revision (hazard ratio [HR], 3.8; 95\% CI, 1.2-16.5; $\mathrm{p}<0.02$ ), reoperation (HR, 2.9; 95\% CI, 1.3-7.4; $\mathrm{p}<0.02$ ), and PJI (HR, 6.4; 95\% CI, 1.2-119.7; $\mathrm{p}<0.03)$. Implant survival rates were $96 \%(95 \% \mathrm{CI}, 92 \%-$ $100 \%)$ and $100 \%$ at 5 years, and $81 \%(95 \% \mathrm{CI}, 70 \%-92 \%)$ and $93 \%(95 \% \mathrm{CI}, 86 \%-100 \%)$ at 10 years for the patients with morbid obesity and those without morbid obesity, respectively $(\mathrm{p}=0.02)$. At 10 years, The Knee Society pain (90 [95\% CI, 88-92] vs 76 [95\% CI, 71-81]; $\mathrm{p}<0.01$ ) and function (61 [95\% CI, 53-69] vs 57 [95\% CI, 42-52]; $\mathrm{p}<0.01$ ) scores were higher in patients with a BMI less than $30 \mathrm{~kg} / \mathrm{m}^{2}$ compared with patients with morbid obesity.

Conclusion Morbid obesity is associated with increased rates of rerevision, reoperation, and PJI after aseptic revision TKA. As the time-sensitive nature of revision surgery may not always allow for patient or comorbidity optimization, these results emphasize the need for improving our care of patients with morbid obesity earlier on during 
the osteoarthritic process. Additional studies are needed to risk stratify patients in the morbidly obese population to better guide patient selection and effective optimization. Level of Evidence Level III, therapeutic study.

\section{Introduction}

The burden of obesity continues to be great, with more than $35 \%$ of Americans now classified as obese $[3,16]$. As the epidemic continues to increase, patients with obesity are of particular concern to arthroplasty surgeons. Obesity not only is associated with an increased risk of knee osteoarthritis, but also with a younger age for primary TKA in patients who are obese than in patients who are not obese [22]. Furthermore, obesity is associated with a higher risk of postoperative complications and need for revision surgery after primary TKA, particularly in patients with morbid obesity $\left(\mathrm{BMI} \geq 40 \mathrm{~kg} / \mathrm{m}^{2}\right)$ [5, 11].

Revision TKA for aseptic failure provides reliable and durable improvement in patient pain and function, with long-term implant survival rates between $80 \%$ and $94 \%$ [2, $7,9,12,20,23,25,26]$. However, to our knowledge, no studies have specifically reported on survivorship or functional scores after aseptic revision TKA in patients with morbid obesity.

The purpose of our study was to evaluate the outcomes of revision TKA performed for aseptic reasons in patients with morbid obesity with those with a BMI less than $30 \mathrm{~kg}$ / $\mathrm{m}^{2}$. Specifically, we aimed to answer the questions: (1) Are patients with morbid obesity at greater risk of repeat revision, reoperation, or periprosthetic joint infection (PJI) compared with patients without obesity after an index revision TKA performed for aseptic reasons? (2) Do patients who are not obese achieve higher Knee Society pain and function scores after revision TKA for aseptic reasons than patients with morbid obesity?

\section{Patients and Methods}

\section{Study Design}

We conducted a single-center, retrospective, 1:1 matched cohort analysis after obtaining approval from our institutional review board. Our institutional total joint registry, which prospectively captures survival data and patient outcomes, was used to identify 1291 both-component index revision TKAs performed for aseptic reasons during a 15year period (1992-2007), including 120 in patients with morbid obesity and 624 in patients with a BMI less than $30 \mathrm{~kg} / \mathrm{m}^{2}$. Exclusion criteria included any prior revision
TKA on the same knee, previous diagnosis of PJI on the same knee, single-component revision, and conversion from a unicompartmental knee arthroplasty to a TKA. We then considered only patients with a minimum of 5 years followup, which was available for $77 \%$ of patients with morbid obesity and $76 \%$ with a BMI less than $30 \mathrm{~kg} / \mathrm{m}^{2}$ $(\mathrm{p}=0.84)$. All $(\mathrm{n}=93)$ patients with morbid obesity were included (morbid obesity group) and matched with a cohort of 93 patients with a BMI less than $30 \mathrm{~kg} / \mathrm{m}^{2}$ (nonmorbid obesity group) using age ( \pm 3 years), date of revision TKA ( \pm 2 years), and sex (Table 1). Primary outcome measures included rerevision surgery (subsequent removal or exchange of femoral and/or tibial components), reoperation for any reason, and subsequent PJI. All diagnoses of PJI met the International Consensus on Periprosthetic Joint Infection criteria [17]. Clinical outcomes were reported for each group, including Knee Society pain and function scores. Patient outcomes were unknown at the time of patient matching.

\section{Patients}

There were 93 patients in each group (morbid obesity and nonmorbid obesity), with each group including 70 (75\%) women. The average age of the patients was 66 years (range, 45-84 years) in the morbid obesity group and 66 years (range, 44-85 years) in the nonmorbid obesity group. There were more patients with Type II diabetes mellitus in the morbid obesity group (25\% vs $11 \%$; $\mathrm{p}<0.02$ ) and more with rheumatoid arthritis in the nonmorbid obesity group $(12 \%$ vs $2 \%$; $p<0.01)$. No patients in either group had Type I diabetes mellitus. There were six (6\%) current smokers in each group. The indications for index revision TKA did not appear to change with time during the study period; the most common reason for revision was aseptic loosening in both groups (Table 1). The average followup was 7.9 years (range, 5-18 years) and 7.3 years (range, 5-16 years) for the morbid obesity and nonmorbid obesity groups, respectively $(\mathrm{p}=0.73)$.

Before revision, PJI was excluded in all knees using serum inflammatory markers and knee aspiration when indicated. Revision TKA was performed using techniques and implants at the discretion of the treating surgeon (Table 2). There were no differences between the two groups with respect to implant constraint, use of stems, type of stem fixation, augments, or the use of adjunctive metaphyseal fixation (sleeves or cones) at the time of revision TKA. Simplex ${ }^{\mathrm{TM}} \mathrm{P}$ cement (Stryker Corporation, Mahwah, NJ, USA) mixed with vancomycin and/or aminoglycoside antibiotics were used in all patients. For each group, a median of $1 \mathrm{~g}$ per batch of vancomycin 
Table 1. Comparison of patient characteristics between groups

\begin{tabular}{|c|c|c|c|}
\hline Factor & Nonobese patients $(\mathrm{n}=93)$ & Morbidly obese patients $(\mathrm{n}=93)$ & $\mathrm{p}$ value \\
\hline Sex: Women (number; \%) & $70(75)$ & $70(75)$ & 0.99 \\
\hline Age (years; range) & $66.0(44-85)$ & $65.6(45-84)$ & 0.73 \\
\hline BMI $\left(\mathrm{kg} / \mathrm{m}^{2} ;\right.$ range $)$ & $26.2(16.4-29.9)$ & $43.3(39.6-59.8)$ & $<0.001$ \\
\hline Followup (years, range) & $7.3(5.0-15.7)$ & $7.9(4.8-18.5)$ & 0.14 \\
\hline Rheumatoid arthritis (number; \%) & $11(12)$ & $2(2)$ & $<0.01$ \\
\hline Current smoker (number; \%) & $6(6)$ & $6(6)$ & 0.99 \\
\hline Diabetes mellitus (number; \%) & $10(11)$ & $23(25)$ & $<0.02$ \\
\hline \multicolumn{3}{|c|}{ Primary reason for revision (number; \%) } & 0.88 \\
\hline Aseptic loosening & $36(39)$ & $33(36)$ & \\
\hline Osteolysis & $17(18)$ & $20(22)$ & \\
\hline Instability & $21(23)$ & $17(18)$ & \\
\hline Stiffness & $8(9)$ & $9(10)$ & \\
\hline Patellar maltracking & $6(7)$ & $7(8)$ & \\
\hline Polyethylene wear & $5(5)$ & $7(8)$ & \\
\hline
\end{tabular}

Table 2. Implant details

\begin{tabular}{|c|c|c|}
\hline Detail & Nonobese (number; \%) & Morbidly obese (number; \%) \\
\hline \multicolumn{3}{|l|}{ Constraint (number; \%) } \\
\hline Posterior stabilized & $25(27)$ & $25(27)$ \\
\hline Varus-valgus stabilized & $63(68)$ & $60(65)$ \\
\hline Hinged & $5(5)$ & $8(9)$ \\
\hline \multicolumn{3}{|c|}{ Femoral support (number; \%) } \\
\hline Augments & $71(76)$ & $75(81)$ \\
\hline Sleeves & $3(3)$ & $3(3)$ \\
\hline Cones & $3(3)$ & $2(2)$ \\
\hline \multicolumn{3}{|l|}{ Tibial support (number; \%) } \\
\hline Augments & $16(17)$ & $14(15)$ \\
\hline Sleeves & $2(2)$ & $4(4)$ \\
\hline Cones & $4(4)$ & $4(4)$ \\
\hline \multicolumn{3}{|l|}{ Stems (number; \%) } \\
\hline Femoral & $89(96)$ & $86(93)$ \\
\hline Tibial & $83(89)$ & $81(87)$ \\
\hline \multicolumn{3}{|l|}{ Stem fixation (number; \%) } \\
\hline Cemented & $82(88)$ & $80(86)$ \\
\hline Press-fit & $11(12)$ & $13(14)$ \\
\hline
\end{tabular}

(range, 0-1 g/batch) and $1.2 \mathrm{~g}$ per batch of aminoglycoside (range, 0-1.2 g/batch) were used.

\section{Statistics}

Descriptive statistics are reported as number (percentage), and continuous variables are reported as mean (standard deviation). Cox proportional regression analysis was performed to assess the association of morbid obesity with the risk of subsequent revision, reoperation, and PJI, with results reported as hazard ratios (HR) with 95\% CI. Multivariate analysis could not be performed owing to the limited number of events, but given the differing prevalences of diabetes mellitus and rheumatoid arthritis between groups, adjusted risk also was assessed to control for the presence of diabetes mellitus and rheumatoid arthritis. Kaplan-Meier survival estimates were used to describe the cumulative probability of the same outcomes, with results reported as a percentage with $95 \% \mathrm{CI}$. 
Log-rank tests were performed to determine statistical significance of survival estimates. Kaplan-Meier estimates were not affected by differences in patient mortality; eight (7\%) patients in the morbid obesity group died at a mean of 11 years after revision, which was similar to $10(11 \%)$ deaths in the nonmorbid obesity group at a mean of 10 years $(p=0.66)$. All statistical calculations were made using JMP ${ }^{\circledR}$ version 9 (Statistical Analysis Software, Cary, NC, USA) with $\alpha$-level set at 0.05 .

\section{Results}

Association of Morbid Obesity with Repeat Revisions, Reoperations, and PJI

We found that patients with morbid obesity were at greater risk of repeat revision, reoperation, and PJI when compared with patients with a BMI less than $30 \mathrm{~kg} / \mathrm{m}^{2}$. Repeat revision was performed in 14 patients (15\%) with morbid obesity compared with three (3\%) who were not obese (adjusted HR, 3.2; 95\% CI, 1.1-14.0; $\mathrm{p}<0.04$ ). An equal proportion of repeat revisions were performed for PJI and aseptic loosening in the morbid obesity group, whereas all repeat revisions were performed for aseptic loosening in the nonmorbid obesity group. Kaplan-Meier estimates for revision-free survival were 96\% (range, 92\%-100\%) at 5 years and $81 \%$ (range, $70 \%-92 \%$ ) at 10 years for the morbid obesity group compared with $100 \%$ at 5 years and $93 \%$ (range, $86 \%-100 \%$ ) at 10 years for the nonmorbid obesity group $(\mathrm{p}=0.02)$ (Fig. 1A). Reoperation for any reason was performed in 24 patients $(26 \%)$ in the morbid obesity group compared with seven $(8 \%)$ in the nonmorbid obesity group (adjusted HR, 2.4; 95\% CI, 1.1-6.2; $\mathrm{p}<0.03)$. Kaplan-Meier estimates for reoperation-free survival were $89 \%$ (range, $83 \%-95 \%$ ) and $71 \%$ (range, $59 \%-83 \%$ ) at 5 and 10 years for the morbid obesity group, and $99 \%$ (range, $98 \%-00 \%$ ) and $85 \%$ (range, $75 \%-95 \%$ ) at 5 years and 10 years for the nonmorbid obesity group $(\mathrm{p}=0.009)$ (Fig. 1B). PJIs also were more frequent in patients with morbid obesity ( $9 \%$ vs $1 \% ; \mathrm{p}<0.02)$, with a trend toward increased risk (adjusted HR, 4.4; 95\% CI, $0.8-82.2 ; \mathrm{p}=0.10$ ) (Table 3). Kaplan-Meier estimates for survival free of PJI were 98\% (range, 96\%-100\%) and $100 \%$ at 5 years and $87 \%$ (range, $77 \%-97 \%$ ) and $100 \%$ at 10 years for the morbid obesity group and nonmorbid obesity group, respectively $(\mathrm{p}=0.04)$ (Fig. 1C).

\section{Morbid Obesity and The Knee Society Scores}

The Knee Society scores (with 95\% CIs) improved after surgery in both groups; however, patients in the nonmorbid
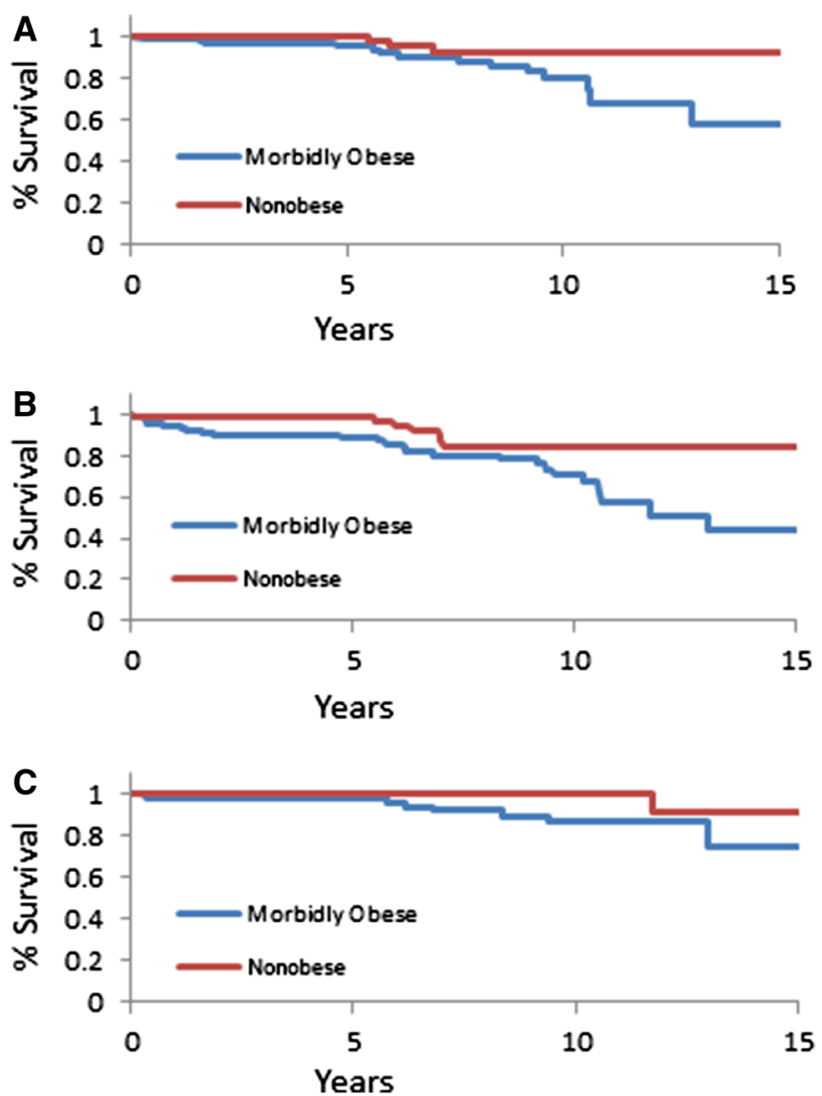

Fig. 1A-C The Kaplan-Meier survival estimates for comparing patients with morbid obesity with those without obesity. (A) The estimated survival free of subsequent revision was $81 \%$ (95\% CI, $70 \%-92 \%$ ) for the patients in the morbid obesity group and $93 \%$ (95\% CI, 86\%-100\%) for patients in the nonmorbid obesity group at 10 years $(\mathrm{p}=0.02)$. (B) The estimated survival free of reoperation was $71 \%$ (95\% CI, 59\%-83\%) for the patients in the morbid obesity group and $85 \%(95 \% \mathrm{CI}, 75 \%-95 \%)$ for patients in the nonmorbid obesity group at 10 years $(\mathrm{p}=0.009)$. (C) The estimated survival free of periprosthetic joint infection was $87 \%$ (95\% CI, 77\%-97\%) for patients in the morbid obesity group and $100 \%$ for patients in the nonmorbid obesity group at 10 years $(\mathrm{p}=0.04)$.

obesity group had better pain and function scores than patients in the morbid obesity group at 2, 5, and 10 years $(\mathrm{p}<0.01)$ (Table 4). At last followup, there were 39 $(42 \%)$ and 13 patients (14\%) with moderate or severe pain in the morbid obesity group, and $37(40 \%)$ and 25 patients (27\%) who reported having either moderate or severe functional limitations in the morbid obesity and nonmorbid obesity groups, respectively.

\section{Discussion}

It is well documented that patients with obesity have more frequent complications and a greater risk of revision after primary TKA $[4,5,14,15]$. As the prevalence of obesity increases, these complications will continue to create 
Table 3. Risk analysis

\begin{tabular}{|c|c|c|c|c|c|c|}
\hline \multirow[t]{2}{*}{ Event } & \multicolumn{3}{|c|}{ Morbid obesity (univariate analysis) } & \multicolumn{3}{|c|}{ Morbid obesity (adjusted for diabetes mellitus and rheumatoid arthritis) } \\
\hline & Hazard ratio & $95 \% \mathrm{CI}$ & $\mathrm{p}$ value & Hazard ratio & $95 \% \mathrm{CI}$ & $\mathrm{p}$ value \\
\hline Revision & 3.8 & $1.2-16.5$ & $<0.02$ & 3.2 & $1.1-14.0$ & $<0.04$ \\
\hline Reoperation & 2.9 & $1.3-7.4$ & $<0.02$ & 2.4 & $1.1-6.2$ & $<0.03$ \\
\hline Deep infection & 6.4 & $1.2-119.7$ & $<0.03$ & 4.4 & $0.8-82.2$ & 0.10 \\
\hline
\end{tabular}

Table 4. Clinical outcome data

\begin{tabular}{|c|c|c|c|}
\hline Outcome & Nonobese group & Morbidly obese group & $\mathrm{p}$ value \\
\hline \multicolumn{4}{|c|}{ Knee Society pain score $(95 \%$ CI) } \\
\hline Preoperative & $58(56-60)$ & $50(47-53)$ & $<0.01$ \\
\hline 2-year followup & $92(90-93)$ & $81(77-85)$ & $<0.01$ \\
\hline 5-year followup & $90(88-92)$ & $76(72-80)$ & $<0.01$ \\
\hline 10-year followup & $90(88-92)$ & $76(71-81)$ & $<0.01$ \\
\hline \multicolumn{4}{|c|}{ Knee Society function score $(95 \% \mathrm{CI})$} \\
\hline Preoperative & $43(39-48)$ & $38(34-42)$ & 0.10 \\
\hline 2-year followup & $72(68-77)$ & $54(49-60)$ & $<0.01$ \\
\hline 5-year followup & $67(61-73)$ & $59(42-55)$ & $<0.01$ \\
\hline 10-year followup & $61(53-69)$ & $57(42-52)$ & $<0.01$ \\
\hline \multicolumn{3}{|c|}{ Pain at most recent followup (number of patients; \%) } & 0.02 \\
\hline None & $44(47)$ & 27 (29) & \\
\hline Mild & 36 (39) & $39(42)$ & \\
\hline Moderate & $13(14)$ & $23(25)$ & \\
\hline Severe & $0(0)$ & $2(2)$ & \\
\hline
\end{tabular}

challenges for arthroplasty surgeons. To date, we have a better understanding of the effect of obesity on the outcome of primary TKA compared with the effect on revision TKA. Watts et al. [24] reported on the outcomes of twostage revision for infection in patients with morbid obesity. In the current study, we evaluated the survivorship and The Knee Society Scores for revision TKA performed in patients with morbid obesity for aseptic reasons. We found that patients with morbid obesity $\left(\mathrm{BMI} \geq 40 \mathrm{~kg} / \mathrm{m}^{2}\right.$ ) had higher rates of subsequent revision, reoperation, and deep infection after aseptic revision TKA when compared with a matched cohort of patients with a BMI less than $30 \mathrm{~kg} / \mathrm{m}^{2}$.

The results of our study should be interpreted in light of certain limitations. Foremost, although our data were prospectively collected, it was examined retrospectively and included only patients in whom the femoral and tibial components were revised. Second, as we practice at a tertiary referral center, many of our patients had prior care at outside institutions, which created a possibility for selection bias. Furthermore, our study was performed at one institution by different surgeons, with reconstruction methods and techniques based on surgeon preference which may have evolved with time. Finally, BMI is inherently associated with systemic comorbidities, which makes it impossible to prove a causal relationship between BMI and outcomes. As an example, there were more patients with diabetes mellitus in the morbid obesity group ( $25 \%$ vs $11 \%$ ) and more with rheumatoid arthritis in the nonmorbid obesity group (12\% vs $2 \%)$. To account for these differences, we reported an adjusted risk analysis that accounted for diabetes mellitus and rheumatoid arthritis, although disease severity was not taken into account. Important variables such as fat distribution, immune suppression, and nutrition status were not quantified.

We found implant survivorship at 5 and 10 years of $96 \%$ and $81 \%$, respectively, in the morbid obesity group compared with $100 \%$ and $93 \%$ in the nonmorbid obesity group. Although there are no data for comparison regarding aseptic revision in patients with obesity, these implant survival rates are better than those reported for patients with morbid obesity after two-stage revision TKA for infection (80\% and 55\% at 5 and 10 years, respectively) [24]. In the current study, morbid obesity was associated with a 3.8 -fold increase in risk of rerevision. This finding is 
consistent with those of a meta-analysis of primary TKAs which showed that patients with a BMI of $30 \mathrm{~kg} / \mathrm{m}^{2}$ or greater had a 1.8-fold increase in the risk of revision surgery compared with patients with a BMI less than $30 \mathrm{~kg} /$ $\mathrm{m}^{2}[11]$.

There is little agreement in the literature regarding the most common reason for failure of revision TKAs $[6,14$, $18,19,21]$. In our study, deep infection was the most common cause of revision and reoperation in the patients with morbid obesity, whereas patients with a BMI less than $30 \mathrm{~kg} / \mathrm{m}^{2}$ were most likely to experience failure of the after revision TKA secondary to aseptic loosening. Mortazavi et al. reported a deep infection rate of $4.4 \%$ after revision TKAs in patients with no prior history of infection [14]. In comparison, $8.6 \%$ of the patients in our morbid obesity group and $1.1 \%$ of the patients in the nonmorbid obesity group had a diagnosis of a deep infection. Although our most common indication for rerevision in patients with morbid obesity was PJI, there also was an increased risk of rerevision for aseptic modes of failure. At the time of repeat revision, most implants included either sleeves or cones for enhanced metaphyseal fixation. Howard et al. [8] and Meneghini et al. [13] reported the respective short-term outcomes for use of femoral and tibial tantalum metaphyseal cones. There was radiographic component incorporation in all patients in their studies. As more longterm reports [10] become available, such techniques may prove to be good options for improved fixation in patients with morbid obesity.

In addition to having worse rerevision, reoperation, and infection rates, patients with morbid obesity had lower clinical outcome scores at all times. These results are comparable to those in a large registry study comparing clinical outcomes after primary TKA among patients in various BMI categories [1]. However, although overall outcomes were lower in our patients with morbid obesity, relative improvement between preoperative and postoperative scores was similar between groups, suggesting that repeat revision is helpful for patients with morbid obesity despite increased associated risks. Denying care to patients with morbid obesity would lead to worse outcomes in pain and function, especially with an already-failed TKA.

The care for patients with morbid obesity and knee arthritis remains challenging. Although there has been substantial contributions to the literature regarding the outcomes of knee arthroplasty in such patients in recent years, surgeons are left without guidance in terms of scientifically modifying treatment $[1,3-5,11,24]$. In turn, surgeons have been left with two options: deny care or continue to operate despite the well-established risks. To meaningfully change the way we treat patients with morbid obesity, future studies first should aim to further riskstratify these patients with potential variables such as associated comorbidities, nutrition, and fat distribution. Once discrete risk factors are identified, we can attempt to meaningfully modify our care and improve patient outcomes.

Revision TKA remains a viable option for patients with morbid obesity and aseptic failure; however, the risks of implant failure, reoperation, and PJI are greater in patients with morbid obesity than those with a BMI less than $30 \mathrm{~kg} /$ $\mathrm{m}^{2}$. The reasons for the increased risk are multifactorial and not yet fully understood. Because revision surgery cannot always wait for patient or comorbidity optimization, our outcomes emphasize the need for improving the care for patients with morbid obesity earlier during the osteoarthritic process. Additional studies are needed to risk stratify patients with morbid obesity to guide patient selection and effective optimization. The poorer outcomes after revision TKAs in patients with morbid obesity should be considered even before the index TKA.

\section{References}

1. Baker P, Petheram T, Jameson S, Reed M, Gregg P, Deehan D. The association between body mass index and the outcomes of total knee arthroplasty. J Bone Joint Surg Am. 2012;94: 1501-1508.

2. Barrack RL, Engh G, Rorabeck C, Sawhney J, Woolfrey M. Patient satisfaction and outcome after septic versus aseptic revision total knee arthroplasty. J Arthroplasty. 2000;15:990-993.

3. Campos P, Saguy A, Ernsberger P, Oliver E, Gaesser G. The epidemiology of overweight and obesity: public health crisis or moral panic? Int J Epidemiol. 2006;35:55-60.

4. Dy CJ, Bozic KJ, Pan TJ, Wright TM, Padgett DE, Lyman S. Risk factors for early revision after total hip arthroplasty. Athritis Care Res (Hoboken). 2014;66:907-915.

5. Friedman RJ, Hess S, Berkowitz SD, Homering M. Complication rates after hip or knee arthroplasty in morbidly obese patients. Clin Orthop Relat Res. 2013;471:3358-3366.

6. Goldberg VM, Figgie MP, Figgie HE 3rd, Sobel M. The results of revision total knee arthroplasty. Clin Orthop Relat Res. 1988;226: 86-92.

7. Haas SB, Insall JN, Montgomery W 3rd, Windsor RE. Revision total knee arthroplasty with use of modular components with stems inserted without cement. J Bone Joint Surg Am. 1995; 77:1700-1707.

8. Howard JL, Kudera J, Lewallen DG, Hanssen AD. Early results of the use of tantalum femoral cones for revision total knee arthroplasty. J Bone Joint Surg Am. 2011;93:478-484.

9. Kasmire KE, Rasouli MR, Mortazavi SM, Sharkey PF, Parvizi J. Predictors of functional outcome after revision total knee arthroplasty following aseptic failure. Knee. 2014;21:264-267.

10. Kamath AF, Lewallen DG, Hanssen AD. Porous tantalum metaphyseal cones for severe tibial bone loss in revision knee arthroplasty: a five to nine-year follow-up. J Bone Joint Surg Am. 2015;97:216-223.

11. Kerkhoffs GM, Servien E, Dunn W, Dahm D, Bramer JA, Haverkamp D. The influence of obesity on the complication rate and outcome of total knee arthroplasty: a meta-analysis and systematic literature review. J Bone Joint Surg Am. 2012;94: 1839-1844. 
12. Mabry TM, Vessely MB, Schleck CD, Harmsen WS, Berry DJ. Revision total knee arthroplasty with modular cemented stems: long-term follow-up. J Arthroplasty. 2007;22(6 suppl 2):100-105.

13. Meneghini RM, Lewallen DG, Hanssen AD, Use of porous tantalum metaphyseal cones for severe tibial bone loss during revision total knee replacement: surgical technique. $J$ Bone Joint Surg Am. 2009;91(suppl 2 pt 1):131-138.

14. Mortazavi SM, Molligan J, Austin MS, Purtill JJ, Hozack WJ, Parvizi J. Failure following revision total knee arthroplasty: infection is the major cause. Int Orthop. 2011;35:1157-1164.

15. Namba RS, Cafri G, Khatod M, Inacio MC, Brox TW, Paxton EW. Risk factors for total knee arthroplasty aseptic revision. $J$ Arthroplasty. 2013;28(8 suppl):122-127.

16. Olshansky SJ, Passaro DJ, Hershow RC, Layden J, Carnes BA, Brody J, Hayflick L, Butler RN, Allison DB, Ludwig DS. A potential decline in life expectancy in the United States in the 21st century. New Engl J Med. 2005;352:1138-1145.

17. Parvizi J, Gehrke T, Chen AF. Proceedings of the International Consensus on Periprosthetic Joint Infection. Bone Joint J. 2013;95:1450-1452.

18. Peters CL, Hennessey R, Barden RM, Galante JO, Rosenberg AG. Revision total knee arthroplasty with a cemented posterior-stabilized or constrained condylar prosthesis: a minimum 3-year and average 5-year follow-up study. J Arthroplasty. 1997;12:896-903.
19. Rand JA, Bryan RS. Results of revision total knee arthroplasties using condylar prostheses: a review of 50 knees. J Bone Joint Surg Am. 1988;70:738-745.

20. Shannon BD, Klassen JF, Rand JA, Berry DJ, Trousdale RT. Revision total knee arthroplasty with cemented components and uncemented intramedullary stems. J Arthroplasty. 2003;18(7 suppl 1):27-32.

21. Stuart MJ, Larson JE, Morrey BF. Reoperation after condylar revision total knee arthroplasty. Clin Orthop Relat Res. 1993;286:168-173.

22. Sturmer T, Gunther KP, Brenner H. Obesity, overweight and patterns of osteoarthritis: the Ulm Osteoarthritis Study. J Clin Epidemiol. 2000;53:307-313.

23. Wang CJ, Hsieh MC, Huang TW, Wang JW, Chen HS, Liu CY. Clinical outcome and patient satisfaction in aseptic and septic revision total knee arthroplasty. Knee. 2004;11:45-49.

24. Watts CD, Wagner ER, Houdek MT, Osmon DR, Hanssen AD, Lewallen DG, Mabry TM. Morbid obesity: a significant risk factor for failure of two-stage revision total knee arthroplasty for infection. J Bone Joint Surg Am. 2014;96:e154.

25. Whaley AL, Trousdale RT, Rand JA, Hanssen AD. Cemented long-stem revision total knee arthroplasty. $J$ Arthroplasty. 2003;18:592-599.

26. Wilke BK, Wagner ER, Trousdale RT. Long-term survival of semi-constrained total knee arthroplasty for revision surgery. $J$ Arthroplasty. 2013;29:1005-1008. 